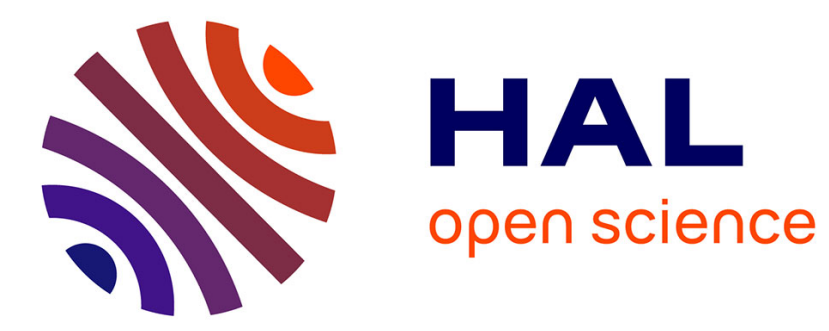

\title{
L'État, de l'appareil à l'apparat
}

Fabien Jobard

\section{To cite this version:}

Fabien Jobard. L'État, de l'appareil à l'apparat. Critique: Studies in Contemporary Fiction, 2012, 780, pp.388-400. hal-00744211

\section{HAL Id: hal-00744211 https://hal.science/hal-00744211}

Submitted on 29 Apr 2015

HAL is a multi-disciplinary open access archive for the deposit and dissemination of scientific research documents, whether they are published or not. The documents may come from teaching and research institutions in France or abroad, or from public or private research centers.
L'archive ouverte pluridisciplinaire HAL, est destinée au dépôt et à la diffusion de documents scientifiques de niveau recherche, publiés ou non, émanant des établissements d'enseignement et de recherche français ou étrangers, des laboratoires publics ou privés. 


\title{
De l'appareil à l'apparat d'État?
}

Pierre Bourdieu, Sur l'État. Cours au Collège de France. 1989-1992, Paris, Seuil, coll. « Raisons d'agir », 2012, 660 p.

L'autorité pédagogique de Bourdieu au Collège de France ne reposait de toute évidence pas sur l'attente suscitée d'un dénouement des questions traitées. Aucun suspense théorique n'est ménagé au seuil des trois années qu'il consacrera à l'État puisque le sociologue annonce dès la première leçon :

\begin{abstract}
Si je devais donner une définition provisoire de ce que l'on appelle «l'État», je dirais que le secteur du champ du pouvoir, qu'on peut appeler «champ administratif » ou «champ de la fonction publique », ce secteur auquel on pense particulièrement quand on parle d'État sans plus de précision, se définit par la possession du monopole de la violence physique et symbolique légitime. J'ai fait, il y a déjà plusieurs années, une addition à la célèbre définition de Max Weber qui définit l'État comme le 'monopole de la violence légitime', que je corrige en ajoutant: 'monopole de la violence physique et symbolique'; on pourrait même dire: 'monopole de la violence symbolique légitime', dans la mesure où le monopole de la violence symbolique est la condition de la possession de l'exercice du monopole de la violence physique elle-même. (p. 14)
\end{abstract}

Cette idée-force est ensuite assénée durant les trois années. «Pour moi, le capital symbolique, c'est le fondement » (p. 327). L'État, est avant tout un stock de «biens symboliques »: à la fois ressources et enjeux de lutte entre les agents qui le forment (p. 127). L'État est même un «principe d'orthodoxie» voire une «théologie» (p. 25). Il administre d'abord, plutôt que des coups de matraque, des catégories de l'entendement; et un État «bien constitué » doit pouvoir, «à la limite », se passer de coercition (p. 239), l'usage de la force physique ne devenant nécessaire que dans les «situations extrêmes» (p. 321). Comme dans les Méditations pascaliennes, la force est conçue comme devant être investie par une «forme de reconnaissance ». Elle ne s'exerce qu'« au-delà de la simple soumission à l'effet physique de la force » (p. 302), si bien que « les rapports de forces sont des rapports de communication » (p. 260). L'appropriation de Weber par Bourdieu altère donc la théorie mère, la notion de « violence symbolique » se substituant ici à celle de «violence physique » située au cœur de la définition sociologique que le savant allemand a donné de l'État.

Pierre Bourdieu suggère pourtant qu'il prolonge, voire achève l'œuvre du père fondateur de la discipline. Max Weber, dit-il, a proposé une définition célèbre de l'État mais il ne l'a pas fait fonctionner, passant rapidement à autre chose. Il reviendrait donc au sociologue français de donner à cette définition sa dimension heuristique propre, d'achever, en quelque sorte, le travail fulgurant - il dit parfois «naif » - de Weber et de ceux qui ont travaillé dans son esprit, comme Norbert Elias ou Charles Tilly, qui font l'objet de longs commentaires. Pour que les corps armés d'un territoire deviennent, avec l'État, une force publique, il faut qu'ils soient au préalable investis d'une croyance collective. Le "monopole de la violence physique » est donc un «monopole de la violence physique et symbolique », c'est-à-dire, in fine, un «monopole de la violence symbolique ». Et «symbolique » ne désigne pas dans ce cas l'ensemble des représentations collectives mais ce qui rend celles-ci possibles et les engendre. Si je remplis un formulaire, explique Bourdieu, c'est que l'État a déposé en moi les structures cognitives qui permettent de penser ce morceau de papier 
comme un formulaire à remplir ; si j'affirme une identité, c'est que je peux m'appuyer sur un opérateur d'État, qui est la carte nationale d'identité (p. 173). Au fondement de l'État se trouve donc une «pensée d'État», violence symbolique déposée dans les esprits et qui agit dans l'ignorance de ceux en qui elle agit.

Le terme-pivot autour duquel tourne cette nouvelle interprétation de Weber est celui de «légitimité ». Chez Weber, la légitimité est une donnée fondamentale de la vie sociale qui se divise en types distincts : traditionnel, charismatique, légal-rationnel. Chez Bourdieu, tout au contraire, la légitimité n'est jamais stabilisée et reste à conquérir. Il n'y a, lorsqu'il s'agit de penser ce qu'est le pouvoir, que des processus et des conflits de légitimation. Dans la sociologie de Bourdieu, les groupes d'une société se livrent ainsi à une lutte incessante pour faire reconnaître leurs avantages acquis comme des différences pouvant fonder une relation d'autorité ou de pouvoir. Dans la substitution du terme «symbolique » au terme «physique » afin de définir l'État, il y a donc plus qu'un simple accomplissement de Weber. La pensée de ce dernier est retournée à la manière dont Marx disait avoir retourné Hegel : en lieu et place d'une légitimité conçue comme établie par le social ou l'histoire, Bourdieu n'a de considération que pour les luttes pour la domination symbolique qui permet l'exercice de la légitimité. Dans sa sociologie, c'est cette lutte qui est le moteur de l'histoire de la société et aussi de l'État.

Au sein de ce cadre théorique général, quelle est la dynamique précise de formation de ces entités politiques appelées «État »? Pour le savoir, il faut d'abord, insiste Bourdieu, défaire ce qu'il appelle la «pensée d'État» —et s'en défaire. Cela passe par deux exigences qui traversent les cours publiés. La première consiste à engager un travail historique permettant d'aller à la source de la production de la violence symbolique et de décrire des formes primitives d'accumulation qui ne sont pas seulement économiques mais concernent d'autres types de ressources, au nombre desquelles les biens symboliques comme le prestige. Ce travail historique n'est cependant pas une tâche d'historien au sens strict, bien qu'il doive être conduit dans l'élément de l'histoire. Il s'agit, insiste Bourdieu, de construire une « génétique de l'État » qui interroge « la genèse des structures objectives » de la société pour restituer «le modèle de la logique de la genèse de l'État» (p. 301). La démarche, qui occupe plusieurs séances, repose ici sur la théorie sociologique des champs construite par Bourdieu, seule à même, selon lui, de reconstituer les mécanismes de reconnaissance et conversion entre ressources accumulées par les différents groupes sociaux ainsi que les relations unissant ou opposant les divers mondes pratiques dans lesquels ces groupes agissent. En ce sens, on l'aura compris, l'histoire de l'État de Bourdieu est schématique et ressemble plus à une théorie sociale du développement de l'État.

Le récit stylisé en serait le suivant. Des «capitaux» sont accumulés par divers groupes parmi lesquels le «capital militaire» (p. 313-317). Devient titulaire du pouvoir politique central celui qui réussit le coup de force ( « coup d'État») de monopoliser ce capital militaire. Un tel geste passe par la dépossession de tous ceux qui usaient jusque là de la force et par la dissuasion de ceux qui seraient tentés d'en user à nouveau. À ce processus de «monopolisation », décrit en détail par Norbert Elias ou Charles Tilly dans l'esprit de Weber, s'ajoute une opération d'« universalisation » qui intéresse particulièrement Bourdieu. Pour être reconnues à l'échelle d'un territoire comme dépositaires d'une force légitime, les fonctions militaires sont ointes d'une force symbolique propre. Suivant un processus de sacralisation, les titulaires du capital de force monopolisé sont peu à peu séparés du monde ordinaire ; leur apparence brute est travestie sous des uniformes, des perruques, des couleurs 
ou bien précédée par des sceaux... Et les luttes autour de ces apparats forment, pour Bourdieu, la matière même de la longue maturation de l'État.

Prenons l'impôt. Voilà, en cette phase de genèse étatique et pendant plusieurs siècles, qui ressemble à un simple racket. Bourdieu le souligne après maints autres ${ }^{1}$. Mais il faut selon lui s'interroger sur ce qui fait que ce racket est perçu comme légitime. Bourdieu insiste alors, non pas sur l'appareil de contrainte, mais sur les apparences des agents et, pour en rester à l'impôt, sur l'importance décisive des livrées, ces uniformes qui devaient assurer l'autorité des personnes chargées de la collecte. D'individus chargés de l'extorsion, ils ont été métamorphosés par une série d'actes de symbolisation, c'est-à-dire de légitimation, en dépositaires reconnus et admis de l'autorité publique.

Le deuxième volet de cette théorie sociale de l'État repose sur la logique des relations entre des champs d'activité et d'intérêt relativement autonomes qui se sont différenciés au fil de l'histoire : la religion, l'armée, le droit, la science, les différents arts puis les différents métiers, etc. Après la phase initiale de monopolisation de la violence légitime, le processus de développement de l'État se saisit dans l'affrontement entre deux «groupes d'agents » principaux : ceux qui doivent la perpétuation de leur domination au principe dynastique des royaumes et des familles de grande lignée et ceux qui aspirent à la domination par valorisation de l'éducation et du mérite attachés à l'étude. En France, «depuis l'origine »c'est-à-dire, chez Bourdieu, depuis le XII ${ }^{\mathrm{e}}$ siècle — ce conflit est le moteur déterminant de la construction de l'État. Pour perpétuer sa victoire sur la noblesse d'épée, la noblesse de robe aurait inventé la compétence scolaire et, avec elle, les valeurs et les instruments qui lui sont liés comme le concours. L'État qu'elle promeut alors est censé servir la neutralité et inculque pour cela un « intérêt à l'universel » jusqu'alors inédit dans l'histoire. Les juristes sont la figure achevée de cette conquête. "Oblats » par excellence du système scolaire de l'âge classique, ils assurent par l'édiction de normes générales à prétentions impersonnelles la toute-puissance de leur compétence et la domination sur tous les autres champs d'activité sociale. Ils parviennent ainsi à imposer l'État nouveau comme un champ au-dessus des autres champs, lieu ultime d'arbitrage des luttes propres à tous les autres univers sociaux.

L'histoire de l'État ne s'achève pas pour autant sur ce triomphe apparent des juristes contre les grandes familles dynastiques, car ces oblats, qui doivent tout au mérite et à l'acquis, tentent par diverses stratégies — déjà exposées dans La Noblesse d'État, ouvrage paru la première année du cours - de refermer les privilèges qu'ils ont obtenus sur euxmêmes et de reconstituer, à leur bénéfice, les logiques patrimoniales et héréditaires de l'État dynastique. Ainsi l'enseignement de Bourdieu sur l'État sonne-t-il aussi comme une mise en garde à l'égard des clercs et leur tentation permanente de voir en l'universel un intérêt partagé et de consacrer leur propre compétence spécifique comme naturelle. L'État est l'objet social, en France du moins, vis-à-vis duquel il est, pour cette raison, le plus difficile pour le savant d'être distancié. Faire tenir ensemble, à son sujet, le propos théorique et l'observation empirique représente un défi (p. 45-47). Il ne peut dès lors qu'être utile, si l'on suit Bourdieu, de soumettre toute sociologie de l'État, y compris la sienne, à une mise à l'épreuve empirique serrée, même si celle-ci ne peut s'appliquer qu'à la colonne vertébrale du raisonnement.

Voyons ainsi ce qu'il en est du côté de l'institution qui se situe au cœur du monopole de la violence : la police, ou encore, dans les termes normatifs modernes, la force publique. L'idée selon laquelle la violence physique nue ne saurait suffire à l'État pour exister en tant 
que tel si elle ne s'accompagne pas d'un ancrage symbolique à même de la soutenir paraît séduisante. L'histoire de la police est en effet l'histoire des modes de légitimation de la force. Un ouvrage-princeps sur l'histoire de la police anglaise, The Politics of the Police, montre abondamment combien le souci premier des gouvernements désireux d'introduire une force publique, payée par l'impôt et comptable auprès de l'autorité politique, fut d'asseoir celle-ci sur le consentement. Cette doctrine du «policing by consent » s'appuyait principalement sur un usage raisonné, autrement dit gradué, de la force ainsi que sur une visibilité au quotidien, laquelle passait essentiellement par l'uniforme. Faisant écho indirectement au propos de Bourdieu qui suggère que la légitimité de l'État est assise lorsque les assujettis imputent l'iniquité ou l'injustice aux fonctionnaires individuels tout en estimant l'État ou le Roi hors de cause, la fameuse Royal Commission on the Police britannique rapportait en 1962 que $25 \%$ à $33 \%$ des citoyens anglais disaient avoir eu de «mauvaises expériences » avec des policiers alors que $82 \%$ à $85 \%$ d'entre eux avaient un "grand respect » à l'égard de la police $^{2}$. Au retour de son exil londonien, Napoléon III avait en tout cas entrepris de fonder une police «à l'anglaise », sur le modèle du «Bobby » que les autorités anglaises avaient exhibé fièrement lors de l'Exposition universelle de Londres en $1851^{3}$. La réflexion porte alors sur l'uniforme. La création du «gardien de la Paix » tient en une couleur, le blanc: gants blancs et bâtons blancs, symboles d'une police propre, comme immaculée, une police de régulation des flux plutôt que de violence sur les corps, qui sera à son tour exportée vers les États soucieux de modernisation à l'occasion de l'Exposition universelle de $1900^{4}$.

Cette blanche histoire n'est pourtant pas la seule à s'écrire. Les policiers parisiens, à la différence des Bobbies londoniens, ont gardé leur épée à la ceinture, après diverses tentatives infructueuses de désarmement qui les avaient amenés à subir l'affront d'une population parisienne moins domestiquée que ce que l'on imaginait. La différence entre l'autorité apparemment naturelle du Bobby londonien et l'autorité contestée du gardien de la paix parisien soulève la question du rapport de la société à l'État, absente des cours de Pierre Bourdieu qui viennent de paraître. L'ordre social et urbain tel qu'il se présentait à Londres dans les années 1820-1860 ne présentait-il pas certains traits susceptibles de rendre acceptable la présence quotidienne d'une police désarmée ? Les historiens n'ont pas tranché le débat (s'il doit jamais l'être) qui consiste à déterminer ce qui rend l'autorité plus aisément «naturelle ». Tout juste se contentent-ils de relever que la police londonienne n'était pas plus légitime à Paris qu'elle ne l'était à New York ${ }^{5}$, ni même que dans les villes du nord de l'Angleterre où les tentatives d'installation d'autorités policières ont souvent viré à l'affrontement brutal avec les populations ouvrières qu'il s'agissait de «civiliser ${ }^{6}$. Le prestige de l'uniforme ne suffit pas également partout à la félicité de l'autorité...

Ces questions ont été examinées par certains historiens qui ont tenté d'embrasser à la fois le développement des institutions policières et leur réception par la société urbaine. Quentin Deluermoz montre ainsi combien l'arrivée du gardien de la Paix en France procède d'une dynamique réciproque d'étatisation de la société et de socialisation de l'État. Le

\footnotetext{
2

3

R. Reiner, The Politics of the Police, Toronto, University of Toronto Press, 1992 ( ${ }^{\mathrm{e}}$ éd.), p. 59.

M. Deflem, Policing World Society, Oxford, Oxford University Press, 1994.

4 Q. Deluermoz, «Circulations et élaborations d'un mode d'action policier. La police en tenue à Paris, d'une police 'londonienne' au 'modèle parisien' (1850-1914) », Revue d'histoire des sciences humaines, 19, 2008 , p. 88.

W. Miller, « Police Authority in London and New York City 1830-1870 », Journal of Social History, 8, 2, 1975, p. 81-101.

Voir les travaux classiques de Richard Storch ( The Plague of the Blue Locusts. Police Reform and Popular Resistance in Northern England, 1840-1857 », International Review of Social History, 20, 1, 1975, p. 61-90 et «The Policeman as Domestic Missionary. Urban Discipline and Popular Culture in Northern England, 1850-1880 », Journal of Social History, 9, 4, 1976, p. 481-509.
} 
nouveau policier ne parvient à établir son autorité à Paris que par son acculturation progressive aux mœurs et aux demandes locales. Voici par exemple à l'œuvre quelques individus qui incarnent et instaurent l'État à cette époque dans le quartier de Belleville :

Les agents prennent leurs repas chez un restaurateur, jouent aux cartes avec les habitués, participent aux micro-réseaux de crédit. Beaucoup pratiquent également une polyactivité (confection de sacs, de bijoux...), mobilisant alors un autre type d'identité sociale [...]. Il semble qu'ils se marient assez facilement dans le quartier, manifestant alors leur pleine intégration. Constamment définis comme policiers, ils sont donc en même temps des personnages frontières ${ }^{7}$.

Comparons maintenant cette observation historique avec un des propos théoriques de Pierre Bourdieu :

Dans une certaine mesure, on pourrait dire que l'intégration - qu'il faut entendre au sens de Durkheim, mais aussi de ceux qui parlaient de l'intégration de l'Algérie, et à laquelle on associe l'idée de consensus - est la condition de la domination. C'est au fond la thèse centrale que je voudrais développer. (p. 352)

Alors qu'il s'agit de la «thèse centrale » du cours, Bourdieu ne consacre pas une heure de peine à ces modalités de réception des actions de l'État et, plus largement, à la disponibilité de la société à son apparat. À l'examen, si l'on suit les travaux sur la pénétration de la force publique dans les sociétés européennes ${ }^{8}$, ce n'est pas l'État qui assoit avec une tranquille assurance la force publique dans des villes «intégrées », mais au contraire les sociétés urbaines qui s'emparent des institutions étatiques à la fois pour les domestiquer et se laisser discipliner par elles. La théorie sociologique de l'État de Bourdieu a donc cette propriété paradoxale qu'elle se déploie avec peu d'égards pour la société.

Tournant le dos à certaines des avancées des sciences sociales de son époque se réclamant pourtant en partie de son enseignement ${ }^{9}$, le sociologue français fond en réalité sa théorie de l'État dans une sociologie des élites, généralisant ainsi le propos de La Noblesse d'État qui portait sur les institutions de production du champ des élites scolaires et sur ses affrontements internes, notamment entre juristes et littéraires, puis entre diplômés de l'ENA et élèves de l'ENS, matrices des cours sur l'État contemporain. Les cours de 1989-1992 prolongent ce point de vue puisque le moteur de l'histoire de l'État reste toujours la lutte entre fractions supérieures de la société. La cécité qui en découle amène souvent à des formulations

Q. Deluermoz, «Présences d'État. Police et société à Paris (1854-1880)», Annales, 64, 2, p. 451.

$8 \quad$ Y compris européennes réelles-socialistes ; voir Th. Lindenberger, « La police populaire de la RDA de 1952 à 1958. Une micro-étude sur la gouvernementalité de l'État socialiste », Annales, 53, 1, 1998, p. 119-152 et F. Jobard, «L'État au quotidien. Histoire de la Volkspolizei », Crime, histoire et société, 9, 1, 2005, p. $139-144$

9

Nous pensons ici notamment au mouvement de «sciences sociales du politique » incarné en France par la revue Politix, créée en 1989, ou à l'« Alltagsgeschichte » allemande, qui examine les modes de domination «par le bas ». Voir par exemple la discussion de la domination chez Bourdieu par Alf Lüdtke in A. Lüdtke (dir.), Herrschaft als soziale Praxis. Historische und sozialanthropologische Studien. Vandenhoeck \& Ruprecht, 1991, p. 29-30 et 42-44 notamment. Cette discussion porte principalement sur l'appropriation/transformation de la domination par les acteurs sociaux. 
fausses, comme avec cette idée que les institutions policières procèdent de l'armée, c'est-àdire d'un processus survenu au cœur des appareils centraux. En réalité, l'écrasante majorité des institutions policières étaient, en Europe et Amérique du Nord, des institutions urbaines et communales. La France n'y fait pas exception, qui dut attendre 1941 pour voir la police centralisée. La doxa selon laquelle la police est dans ce pays une institution « régalienne » relève bien de la «pensée d'État» et prend ainsi en défaut même ses contempteurs les plus résolus. L'exemple des juristes est tout aussi éloquent pour souligner le biais élitiste de Bourdieu. Ceux dont il traite sont en effet les juristes de Cour et des institutions les plus prestigieuses, comme le Parlement de Paris. Le travail minutieux d'élaboration des normes relatives à l'étiquette, à la couleur des étoffes, lorsque le Parlement se réunit, lorsque ce Parlement reçoit le Roi, lorsqu'il envisage de lui adresser des remontrances, etc., suscite de longues pages admiratives du sociologue qui retrouve là une logique sociale de clôture d'un champ spécifique d'activité sur lui-même. Mais il n'a pas d'intérêt pour la foule des juristes qui, petites mains du droit, élaborent le pouvoir réglementaire qui régit pourtant le quotidien des ressortissants d'un État ${ }^{10}$.

Ce pouvoir - la «police administrative » en langage savant - ainsi que les normes qu'il engendre, Paolo Napoli les a décrits comme « une anomalie du point de vue de la rationalité juridique ${ }^{11}$. Car le pouvoir réglementaire s'intéresse à la circulation des hommes, des biens, des bêtes, mais aussi de la monnaie et des idées, bref, au marché et à la rue. S'il représente une anomalie, c'est qu'il relève de la souveraineté tout en étant affranchi du Prince. Qu'il s'agisse de l'ordonnance de 1388 sur « l'entretien de la voirie de Paris », prise dans un souci de «bon gouvernement et état de la police» ou bien de celle de 1350 sur les «sansaveux $»^{12}$, le souverain aspire à régir la vie concrète des hommes et, dans le même mouvement, il se rend comptable d'efficacité, de bon ordre et de respect de la justice « ordinaire ». Il ne s'affirme donc pas seulement par sa capacité cérémonielle et sa puissance symbolique, mais par son aptitude à assurer le maintien d'un ordre conforme au sentiment de justice qui anime les collectivités locales. Non pas, donc, magnificence de l'apparat et luttes d'étoffes, mais conquête par le souverain de son pouvoir politique dans l'aptitude à voir ses actes adoptés, compris, domestiqués par les sociétés urbaines ou villageoises.

$\mathrm{Du}$ point de vue de ce droit réglementaire par lequel l'État ou le souverain s'établissent, la «raison d'État» dont il est question dans les cours de Bourdieu prend un visage plus précis. Chez ce dernier, elle naît de la bureaucratie qui s'autonomise par rapport à la «maison du Roi» lorsque l'appareil de domination se dépouille de ses structures dynastiques. Vecteurs de cette transformation, les juristes produisent la «doxa» nouvelle nécessaire à un ensemble inédit de règles qui ne sont pas celles du monde ordinaire. Si dans le monde ordinaire, explique Bourdieu, «il faut être gentil avec ses parents, il faut soutenir ses enfants, etc. Au contraire, [il est connu que] "l'administration ne fait pas de cadeaux" » (p. 407). L'examen de l'activité des juristes qui travaillent au service de la consolidation de la souveraineté du Roi, montre pourtant une réalité qu'il ignore: celle d'une autorité réglementaire prise dans les linéaments du social, des ordres urbains ou villageois. La « raison d'État » peut bien ressembler à l'ensemble des doctrines baroques sur la prise et la

\footnotetext{
10 On est étonné, là encore, compte tenu de la théorie sociologique du droit qu'il avait présentée dans «La force du droit», Actes de la recherche en sciences sociales, 64, 1986, p. 3-19, tout entier consacré aux logiques gouvernant « la division du travail juridique » et aux positions polarisées des juristes, du haut magistrat de la Cour de cassation à la juge aux affaires familiales d'un tribunal d'instance et au juriste d'entreprise.

11 P. Napoli, Naissance de la police moderne. Pouvoir, normes, société, Paris, La Découverte, 2003 , p. 9.

12 Ibid., p. 21. Voir aussi F. Martin, Justice et législation sous le règne de Louis XI, Paris, LGDJFondation Varenne, 2009.
} 
conservation du pouvoir ${ }^{13}$, elle exprime aussi le devoir de «traduire le principe juridique de sa souveraineté dans une série d'actions efficaces sur les hommes et les choses ${ }^{14}$.

De même, dans sa reconstruction de l'histoire de l'impôt, le sociologue n'est pas assez attentif à ce qu'il en fut de l'exercice concret du pouvoir, ici de la collecte. Car, en France, l'administration fiscale dut composer avec la demande sociale et les exigences de justice :

Les agents de l'administration, écrit Nicolas Delalande, sont donc appelés, à tous les échelons hiérarchiques, à se référer à des conceptions de la justice fiscale, non écrites dans la loi, pour décider s'ils doivent ou non accepter les réclamations des contribuables (...). L'application des lois fiscales suppose un ensemble d'écarts et d'arrangements avec la norme qui rendent possible le bon déroulement du prélèvement (...). Les percepteurs doivent se montrer à l'écoute des contribuables, se faire apprécier d'eux et manier avec habileté les ressources de pédagogie et d'adaptabilité que leur hiérarchie leur conseille d'utiliser ${ }^{15}$.

On pourrait multiplier les exemples convergents, comme celui aussi de la carte d'identité et de ses usages, qui montreraient qu'il ne suffit pas que l'État édicte pour que les agents souscrivent ${ }^{16}$. Bourdieu a développé une théorie de l'État qui, bien que fondée sur la «violence symbolique » ou les ressorts de l'obéissance, ne porte guère d'attention précise aux formes par lesquelles ce consentement est réalisé. Ce qui se donnait au départ pour un examen de l'apparat reste attaché à l'analyse de l'appareil et des luttes qui le traversent ${ }^{17}$.

Si l'on s'accorde sur ces limites du pari théorique proposé par Bourdieu, de quelle nature peut bien alors être l'audace qu'il revendique lorsqu'il entend dévoiler le substrat «symbolique »du monopole de la domination «physique » que Weber aurait considéré comme l'essence même de l'État ? En fait, si la démarche du sociologue français rompt ici avec celle de Weber, ce n'est pas nécessairement au sens où il le suggère. D'abord, lorsqu'il s'intéresse aux modalités par lesquelles la légitimité est escomptée, c'est au prix d'une indifférence à ses conditions d'effectivité et de réception. En cela, Bourdieu s'inscrit au fond dans la droite ligne de Weber qui, lui aussi, selon le relecture qui en est faite aujourd'hui, ne «se préoccupe aucunement de rendre comte des logiques motivationnelles qui président à l'obéissance des dominés ${ }^{18}$. On sait mieux à l'heure actuelle que ce désintérêt de Weber était dû à ce que sa définition ne rompait au fond avec la conception dominante de l'État par les juristes de son époque qu'en y ajoutant l'idée de légitimité, celle du monopole de la

13

L. Marin, « Pour une théorie baroque de l'action politique », in G. Naudé, Considérations politiques sur les coups d'Etat, Les éditions de Paris, 1988, en part. p. 22-25 sur l'autonomisation de l'Etat par l'opération du secret et de la dissimulation.

14 P. Napoli, op. cit., p. 28.

15 N. Delalande, «Le consentement à l'impôt en France : les contribuables, l'administration et le problème de la confiance », Revue d'histoire moderne et contemporaine, 56, 2, 2009, p. 151-152.

16 L'identité des individus ne se réduit pas au simple document estampillé malgré tous les cérémoniels dont on le pare. Voir E. Ribert, Liberté, égalité, fraternité. Les jeunes issus de l'immigration et l'appartenance nationale, Paris, La Découverte, 2006.

17 Il faut toutefois noter que Bourdieu devait être conscient de l'orientation prise par son enseignement puisqu'il déclare à ses auditeurs, le 21 novembre 1991: «Au fond, je ne savais pas ce que je faisais, je voulais décrire la genèse de l'État et en réalité je décris la genèse du champ du pouvoir, c'est à dire l'espace différencié à l'intérieur duquel des détenteurs de pouvoirs différents luttent pour que leur pouvoir soit le pouvoir légitime ».

18 C. Colliot-Thélène, «La fin du monopole de la violence physique légitime ? », Revue d'études comparatives est-ouest, 34, 1, 2003, p. 27. 
violence physique étant alors déjà communément admise ${ }^{19}$. Ainsi, la rupture mise en avant par Bourdieu avec l'idée que la violence physique est le substrat de l'État était-elle déjà celle de Weber contre ses maîtres.

Reste donc à déterminer en quoi ce terme ajouté peut transformer la nature du monopole dont l'État est le produit autant que l'expression. Catherine Colliot-Thélène défend l'idée selon laquelle, sous la plume de Weber, le «monopole de la violence physique légitime » était en réalité le monopole du droit à exercer la violence ${ }^{20}$. Si l'on suit cette lecture, Weber ouvrait la voie à une analyse de l'État en l'espèce négligée par Bourdieu : la capacité de l'Etat à protéger, y compris par la violence physique, les droits individuels. La substitution de la catégorie de «violence symbolique » à celle de «violence physique », qui traverse les cours sur l'État du grand sociologue français, permettra certainement de mieux comprendre la concurrence entre les groupes dominants et les formes symboliques de la domination. Mais elle obscurcit les perspectives ouvertes par Weber en refermant définitivement l'État sur lui-même.

Fabien Jobard

19 J. Q. Whitman, «Aux origines du 'monopole de la violence' », dans C. Colliot-Thélène, J.-F. Kervégan (dir.), De la société à la sociologie, Lyon, Ecole normale supérieure, 2002, notamment p. 73-85.

20 C. Colliot-Thélène, art. cit., p. 16. 